\title{
Implementation of New Congestion Control Mechanism in Manet Using Buffer Management
}

\author{
Prachi Jain $^{1}$, Vijay Prakash ${ }^{2}$ \\ SVITS, Indore
}

\begin{abstract}
A number of routing algorithms have been established to facilitate routing). However, most algorithms just implement the sh ortest path routing and do not follow well to MANET characteristics, such as the belief and battery levels of the mobile nodes, critically $i$ nfluence the suitability of the routing decision. Network based congestion avoidance which involves handling the queues in the network devices is an integral part of any network. Maximum of the mobile networks use Drop tail queue management where packets are droppe d on queue overflow which is global synchronization problem. This Paper proposes a new scheme of buffer management to control cong estion in MANET. We set a buffer from sender to receiver with an $X(X$ is not defined yet) buffer limit. There are several neighboring no des around that main buffer and every node have its own limit for placing no. of packets in it so that packets should not be dropped.
\end{abstract}

Keywords: Mobile Ad hoc Network, Congestion Control, Buffer Management, Neighboring nodes, Packet drop

\section{Introduction}

A Mobile Ad hoc Network (MANET) is a category of ad ho $\mathrm{c}$ network. Ad hoc means arranged or happening whenever $\mathrm{n}$ ecessary and not planned in advance. Ad hoc is a Local Area Network which permits new network devices to be added sp eedily. Mobile ad hoc network is a group of independent nod es which forms impermanent network without any immobile infrastructure or central manager. For establishing network wireless connections (wi-fi) are used or any additional medi um such as satellite or cellular transmission. Each and every device in a MANET is permitted to move autonomously in a ny path. The movement of nodes is casual in MANET. So M ANETs have a dynamic topology.

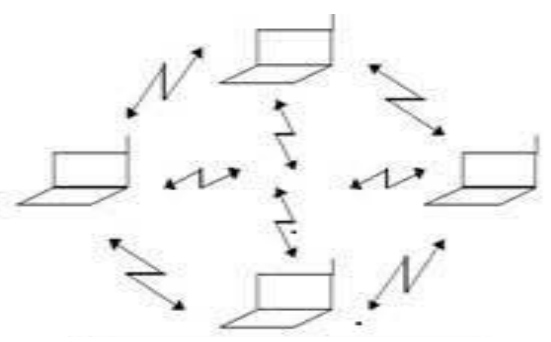

Mobile Adhoc Network

MANET is a communication medium in daily human life an $\mathrm{d}$ applications zones of MANET are growing rapidly Conges tion control and securities are major tasks in MANET. Cong estion control works very well in TCP over internet. But due to dynamic topology congestion control is a challenging tas $\mathrm{k}$ in mobile ad hoc network. Many approaches have been pla nned for congestion control in MANET. Congestion control technique is the procedure by which the network bandwidth is dispersed across multiple end to end connections. A conge stion control scheme on the other hand, MANET nodes are $r$ esource controlled devices which have limited battery life an $\mathrm{d}$ memory/storage spaces. In such circumstances, data sent fr om sources which spread packets with a smaller amount of $d$ ata rates does not get a fair part in queues. Though, such sou rces are not accomplished to convey packets with higher am ount of data rates due to contention of nodes and interfering areas on the path. Taking it as an benefit, some other nodes $b$ ehave belligerently as they can send packets with higher data rates to the end node through intermediate nodes. Therefore, they can inhabit larger space in the queues in unfair manner.
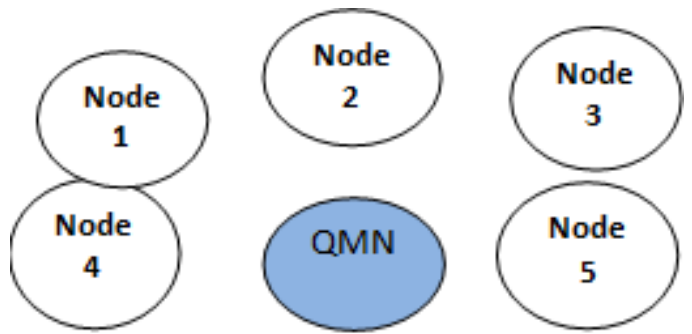

Considered MANET Scenario

\section{Existing Queue Management Schemes}

Congestion control involves the design of mechanisms and a lgorithms to statistically bind the demand-capacity incongrui ty or dynamically control traffic sources when such incongru ity arises. It has been exposed that static results such as alloc ating more buffers, providing faster links or faster processor $\mathrm{s}$ are not in effect for congestion control purposes.

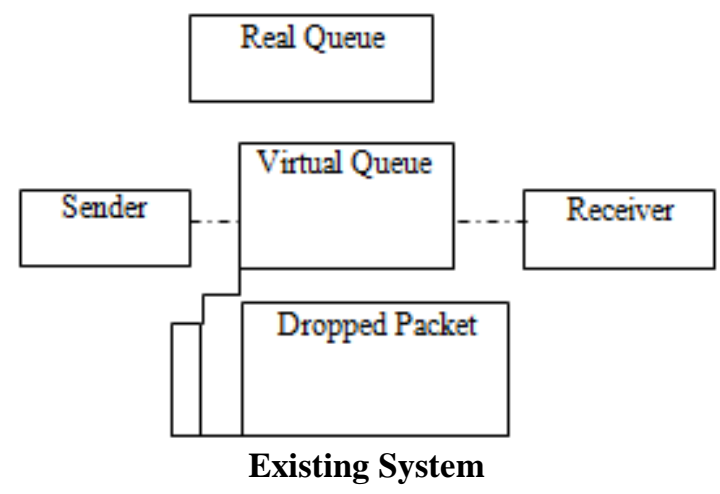

In high-speed networks with connections with high delay-ba ndwidth products, gateways are possible to be aimed with co ngruently large maximum queues to accommodate transient congestion. In the present Internet, the TCP transport protoc ol senses congestion only after a packet has been dropped at the gateway. However, it would obviously be unwelcome to

\section{Volume 4 Issue 11, November 2015}




\section{International Journal of Science and Research (IJSR) \\ ISSN (Online): 2319-7064 \\ Index Copernicus Value (2013): 6.14 | Impact Factor (2014): 5.611}

have large queues (possibly on the order of a delay-bandwidt $\mathrm{h}$ product) that were filled much of the time; this would kno wingly increase the average postponement in the network. $\mathrm{T}$ herefore, with progressively high-speed networks, it is progr essively important to have mechanisms that have throughput high but average queue sizes small. RED measures congesti on by queue length. Importantly, the choice of congestion $\mathrm{m}$ easure finds how it is updated to replicate congestion. To ev ade congestion the router has to drop packets, before the situ ation has developed hopeless, the idea is that there is time fo $r$ action to be taken before it is too late. To find when to start discarding, uphold a running average of their queue distance on some line surpasses threshold the line is said to be conge sted and starts discarding. Second, recognize a possibility pu rpose which is a piecewise linear and increasing purpose of $t$ he congestion measure. Finally, the congestion information i $\mathrm{s}$ carried to the users by either dropping a packet or setting it $\mathrm{s}$ ECN bit probabilistically.

REM is an active queue management scheme that aims to att ain both high use and small loss and delay in a simple and sc alable manner. The first idea of REM attempts to match user rates to network size while clearing buffers, regardless of nu mber of users. The second idea implants the sum of link pric es (congestion measures), summed over all the routers in the path of the user to the end-to-end marking (or dropping) pos sibility. Number of active flows shares a linear relationship with number of dissimilar flows in the buffer. Adaptive Virt ual Queue (AVQ) is designed that results in low-loss, low-de lay and high utilization operation at the link. AVQ algorithm upholds a virtual queue whose capacity is less than the actu al capacity of the link. When a packet reaches in the real que ue, the virtual queue is also updated to reflect the new packet $\mathrm{s}$ in the real queue to replicate the new entrance. Packets in $\mathrm{t}$ he real queue are marked/dropped using ECN mechanism w hen the virtual buffer overflows.

\section{Literature Review}

Muhammad Aamir et.al.[2013] make known to a new sche me of buffer management to handle packet queues in Mobile Ad hoc Networks (MANETs) for immobile and mobile nod es. In this scheme, try to achieve efficient queuing in the buf fer of a centrally interactive MANET node through an active queue management strategy by assigning dynamic buffer $\mathrm{sp}$ ace to all neighboring nodes in amount to the number of pac kets received from neighbors and hence controlling packet $d$ rop probabilities. the suggested algorithm is generated on the occurrence of a selected incident, the allocation is dynamica lly adjusted according to the rapid share of neighbors in the node's buffer and the gap between the occupied and allotted buffer space.

Iyyapillai Ambika et. al.[2014] his research proposes an eff ective queuing architecture, which supports both elastic and inelastic traffic. The packets of inflexible flows are always st ored ahead of those of the elastic flows. If a link is critically loaded by the inflexible traffic, it results in large delays and elastic traffic may have some postponement restrictions that are no eligible. Based on PID mechanism, priority dropping active queue management algorithm (PID_PD) provides the illustrious service for the dissimilar layers or frames accordi ng to their priority. Simulation outcomes proved that the pro posed design offers better fairness and delivery ratio with re duced postponement and drop.

Mr. A. Chandra et. al. [2014] this paper completes an effor $\mathrm{t}$ to present a queue management approach. However the met hod has outdone present queue management techniques RE $\mathrm{D}$ and REM. Here choke packet mechanism is used to send $\mathrm{t}$ he response to sender. It involves extra overhead to the traffi c. Maintenance of virtual queue consumes additional buffer space. Reducing of the size of virtual queue can be carried in future.

Pham and Perreau et. al. [2003] have proposed a load-bala ncing mechanism that impulses the traffic farther from the $\mathrm{c}$ enter of the network, using a routing metric that takes into ac count a node's degree of importance, for both proactive and reactive routing protocols. Their method progresses the load distribution and significantly enhances the network performa nces in terms of average delay and dependability. Though thi s method use only single path routing, which may cause extr a overhead under high node mobility due to frequent route br eaks.

\section{Proposed System}

We propose a scheme of buffer management for packet queu es in MANETs for immobile and mobile nodes. For a MAN ET node, the packet queue is maintained in such a way that a $\mathrm{n}$ equal buffer space is assigned to each neighboring source a nd an allowable extension is also available to each neighbor to avoid any underutilization of resources. The apportionme $\mathrm{nt}$ is made in the buffer of a centrally communicating MAN ET node and it is created on number of packets received in $t$ he queue at node's buffer to utilize the buffer space efficientl $\mathrm{y}$ without any takeover of some surrounding source. We con sider a MANET model working on Ad hoc On demand Dista nce Vector (AODV) routing protocol. It is a responsive prot ocol in which sources get routes to destinations when they $\mathrm{d}$ emand for the same. Nodes only recognize their neighbors th rough routing table entries and keep track of neighbors by ex changing HELLO packets periodically.

This research paper proposes a new scheme of buffer manag ement to control congestion in MANET. We set a buffer fro $\mathrm{m}$ sender to receiver with an $\mathrm{X}(\mathrm{X}$ is not defined yet) buffer $\mathrm{li}$ mit. There are several neighboring nodes around that main $b$ uffer and every node have its own limit for placing no. of pa ckets in it. When the packets are send from sender then firstl $y$ it goes to neighboring nodes and then one by one send to main buffer and from main buffer packets are send to destina tion. In this whole process, packets are not dropped or discar ded. Every node will have its own sequence number so to av oid congestion.

\section{Volume 4 Issue 11, November 2015}




\section{International Journal of Science and Research (IJSR) \\ ISSN (Online): 2319-7064}

Index Copernicus Value (2013): 6.14 | Impact Factor (2014): 5.611

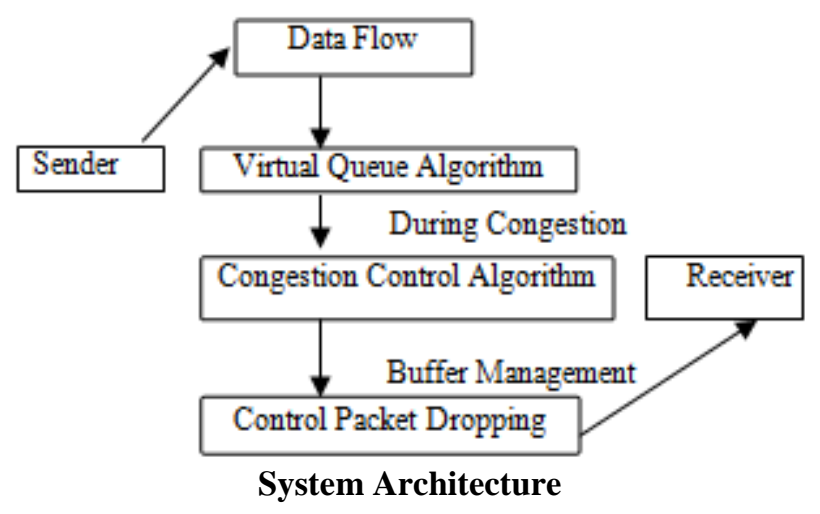

\section{Result \& Discussion}

Screenshot 1 In the screenshot shown below, when we run $t$ he program then the first page open is shown below contains five points:

1. Draw Nodes

2. Set Source and Destination

3. Send Data

4. Graph

5. Clear

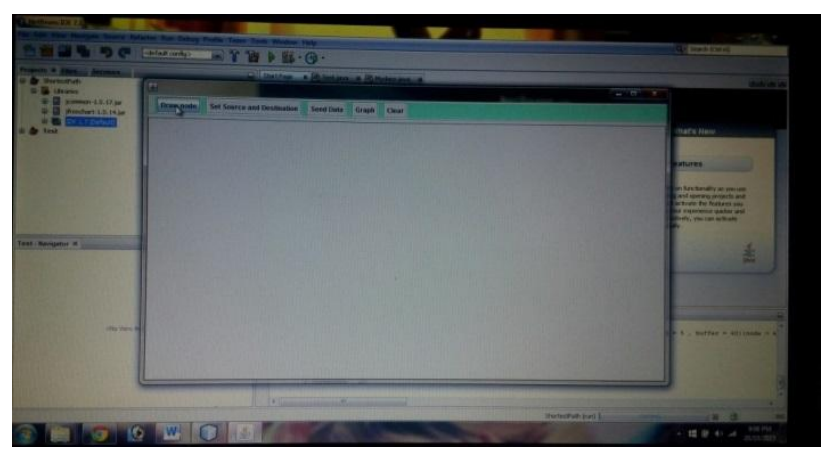

Screenshot 1

Screenshot 2 In the screenshot shown below, when we click on draw nodes option then a dialogue box get open which $\mathrm{w}$ ill ask for no. of nodes.

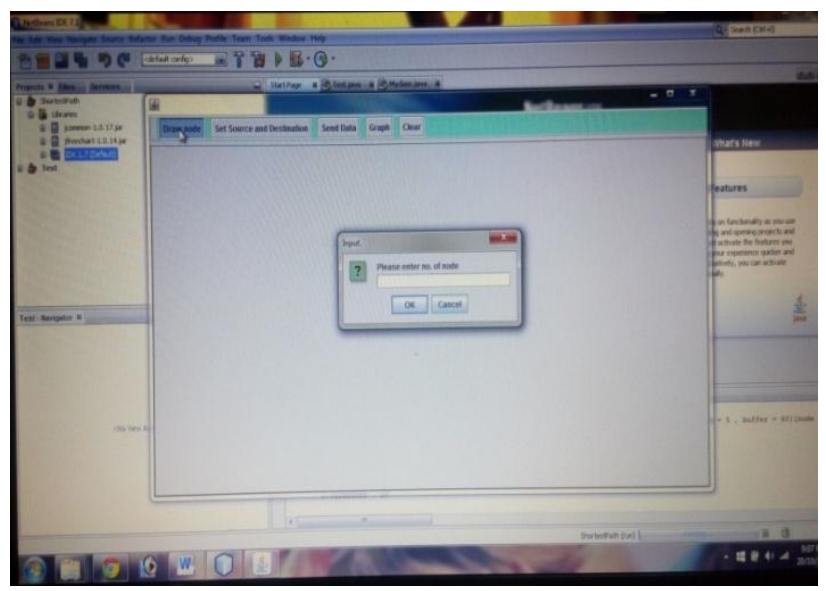

Screenshot 2

Screenshot 3 In the screenshot shown below, we enter no of nodes according to us. As here in this screenshot no of node s entered are 20.

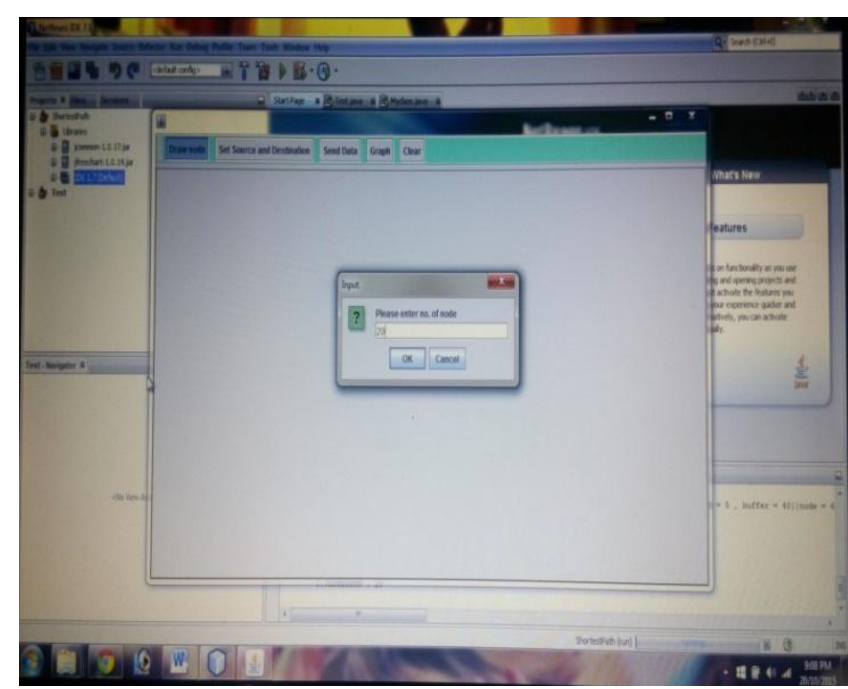

\section{Screenshot 3}

Screenshot 4 In the screenshot shown below, no of nodes en tered is drawn.

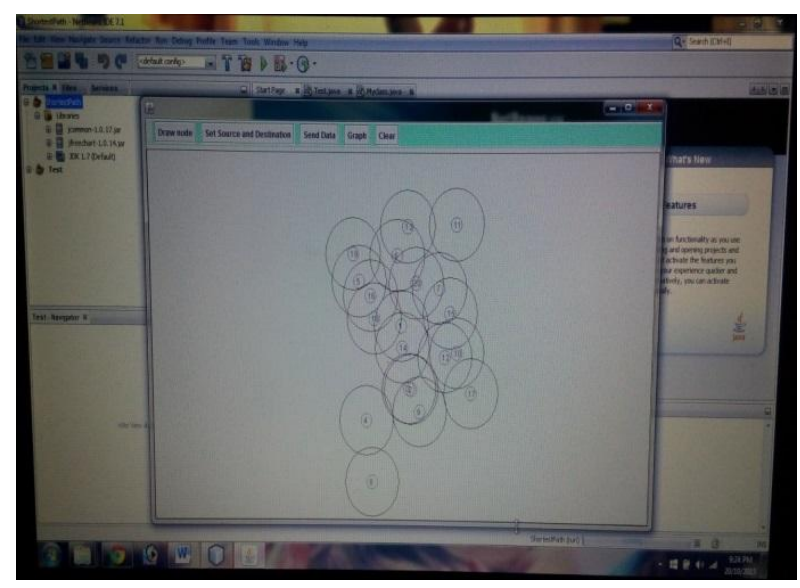

Screenshot 4

Screenshot 5 In the screenshot shown below, source nodes a re entered. Here in this screenshot source node entered is 8 .

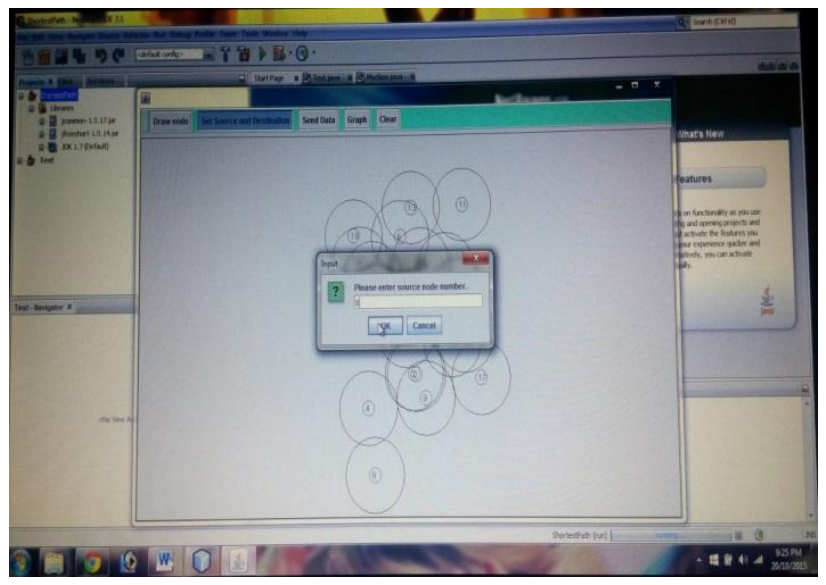

Screenshot 5

Screenshot 6 In the screenshot shown below, a destination $n$ ode is entered. Here in this screenshot destination node enter ed is 11 . 
International Journal of Science and Research (IJSR)

ISSN (Online): 2319-7064

Index Copernicus Value (2013): 6.14 | Impact Factor (2014): 5.611

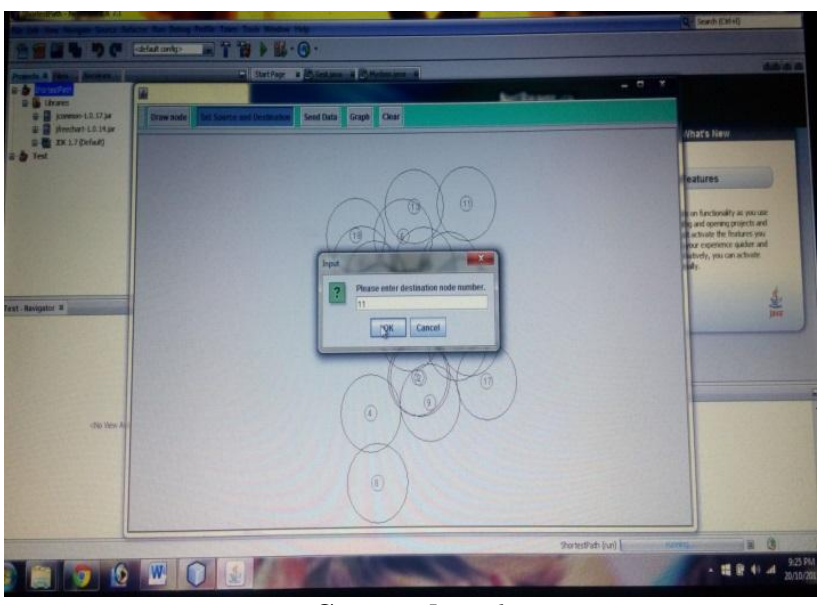

Screenshot 6

Screenshot 7 In the screenshot shown below, shortest path $\mathrm{f}$ rom source to destination is determined. Here in this screens hot the shortest path is $8,4,3,2,12,7,11$.

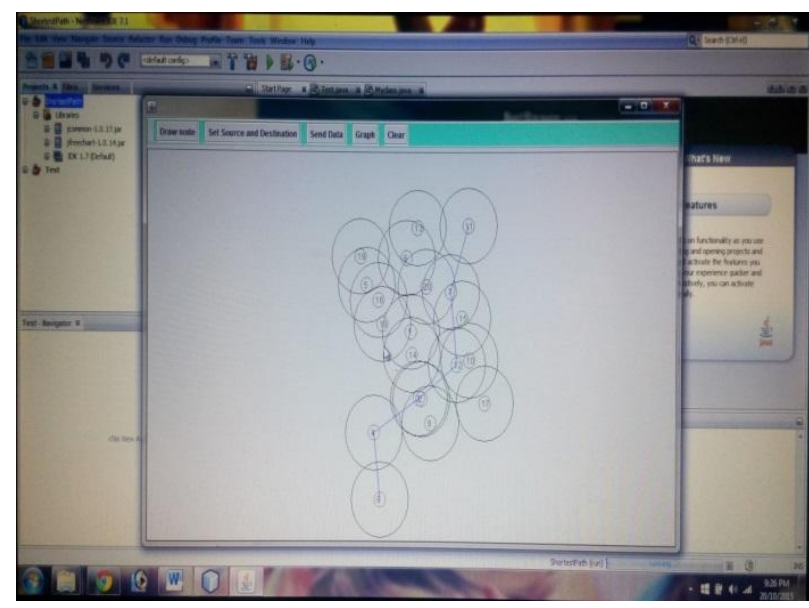

Screenshot 7

Screenshot 8, 9, 10, 11, 12In the screenshot shown below, tr ansfer of data node by node is shown. Firstly data transferre $\mathrm{d}$ from source node 8 to node 4 then from node 4 to node $3 \mathrm{t}$ hen from node 3 to node 2 then from node 2 to node 12 then from node 12 to node 7 then finally from node 7 to destinati on node 11 .

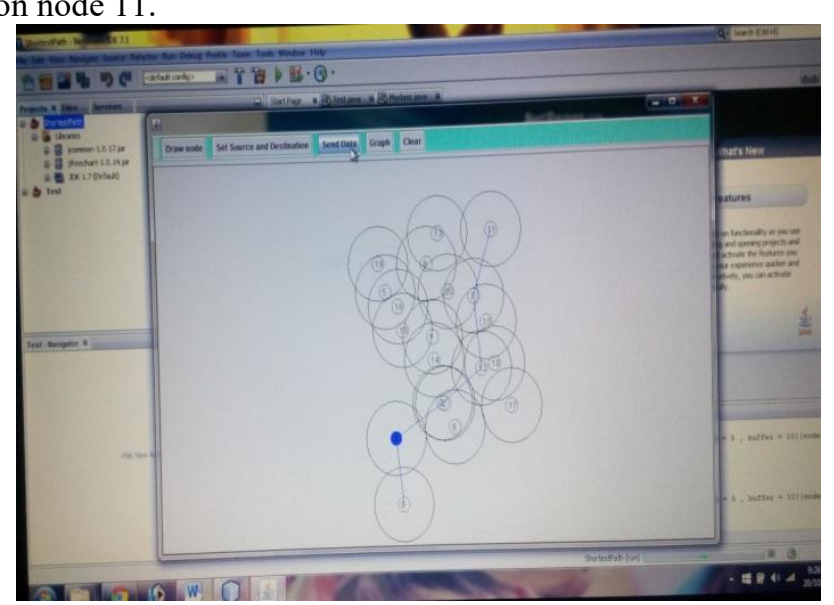

Screenshot 8
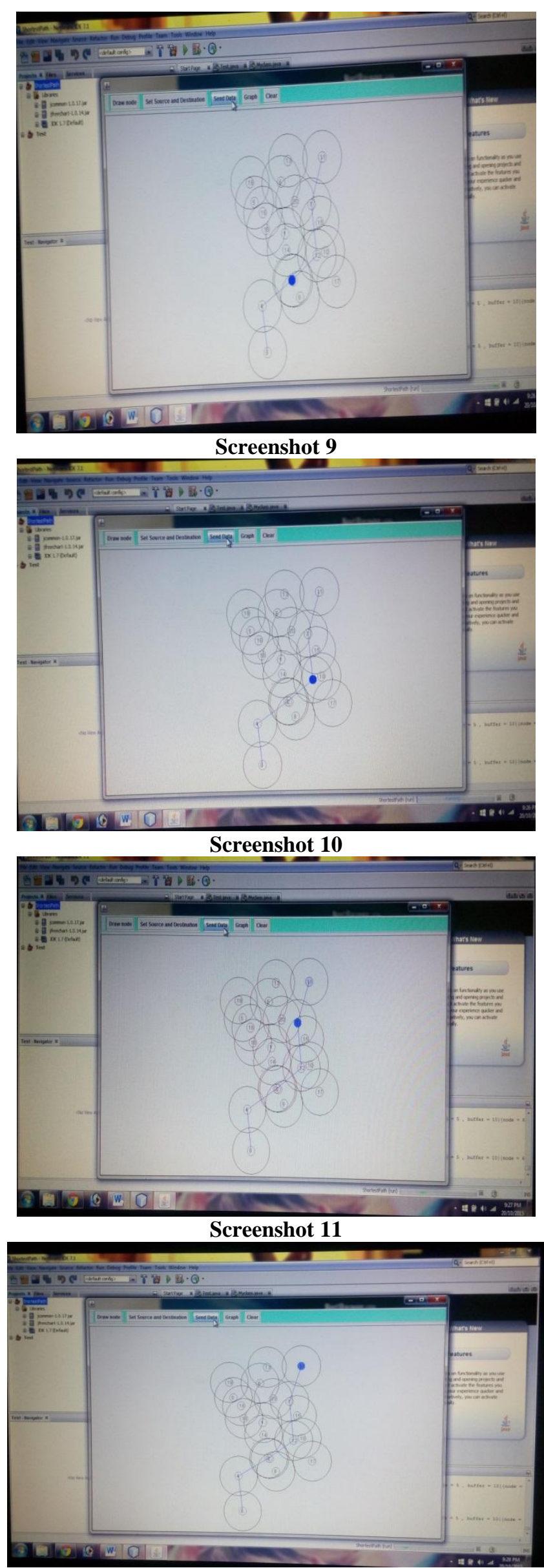

Screenshot 12

Volume 4 Issue 11, November 2015 www.ijsr.net 


\section{International Journal of Science and Research (IJSR) \\ ISSN (Online): 2319-7064}

Index Copernicus Value (2013): 6.14 | Impact Factor (2014): 5.611

Screenshot 13 In the screenshot below, a comparison of tim e vs no of nodes graph is shown.

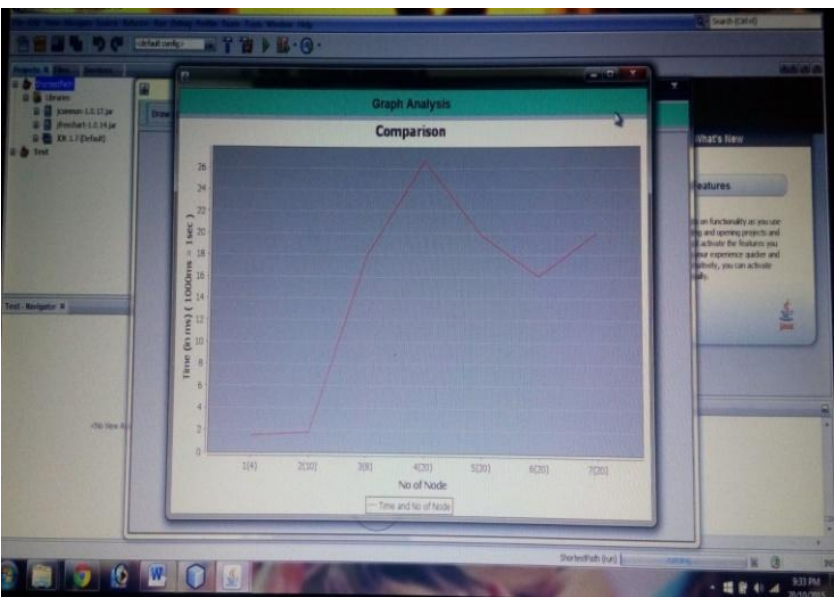

Screenshot 13

\section{Conclusion}

As well as also we presents an overview on congestion contr ol algorithms. We can conclude that there is no single algorit $\mathrm{hm}$ for congestion control in mobile ad hoc network. Nodes $\mathrm{i}$ $\mathrm{n}$ MANET have limited bandwidth, buffer space, queue etc. So it is essential to distribute the traffic among the mobile no des. In MANET, to improve the performance, it is very esse ntial to balance the traffic congestion. Main objective of any congestion control algorithm is to balance the traffic to incre ase throughput of the network. Also it is possible to maximiz e nodes transfer, packet delivery ratio, and minimizes traffic congestion, end-to-end packet delay and network performan ce can be improved. In our future work we will propose mult ipath load balancing with queue scheme as well as acknowle dgment delay difference base estimation technique, also find out number of data drop from the network with reason that work help to rectification of our work and minimize the con gestion from the network.

\section{References}

[1] M. Aamir, M. Zaidi, and H. Mansoor, Performance analysis of Diffserv based quality of service in a multimedia wired network and VPN effect using OPNET, International Journal of Computer Science Issues, vol. 9, no. 3, pp. 368-376, 2012.

[2] L. Chrost and A. Chydzinski, On the evaluation of the active queue management mechanisms, in Proc. $1^{\text {st }}$ Int. Conf. On Evolving Internet (INTERNET), 2009, pp. 113-118.

[3] K. R. S. Floyd and D. Black, The addition of explicit congestion notification (ECN) to IP, RFC 3168, Sep. 2001.

[4] P. G. Kulkarni, S. I. McClean, G. P. Parr, and M. M. Black, Proactive predictive queue management for improved QoS in IP networks, in Proc. IEEE ICN/ICONS/MCL 2006, 2006.

[5] S.T. Hasson, E. Fadil, Queuing approach to model the MANETs performance. Br. J. Sci. 6, 18-24 (2012)

[6] Y.-F. Guo, G.-S. Kuo, A packet scheduling framework for multipath routing in mobile ad hoc networks, in Vehicular Technology Conference (IEEE, 2007), pp.
233-237

[7] R. Patil, A. Damodaram, R. Das, Cross layer fair scheduling for MANET with 802.11 CDMA channels, in First Asian Himalayas International Conference, IEEE (Kathmandu, 2009), pp. 1-5

[8] T. Salonidis, L. Tassiulas, Distributed on-line schedule adaptation for balanced slot allocation in wireless ad hoc networks, in Quality of Service, Twelfth IEEE International Workshop (IEEE, 2004), pp. 20-29

[9] Y. He, R. Yuan, J. Sun, W. Gong, Semi-random backoff: towards resource reservation for channel access in wireless LANs, in 17th IEEE International Conference on Network Protocols (IEEE, Princeton, 2009), pp. 2130

[10] H. Xiao, W.K.G. Seah, A. Lo, K.C. Chua, A flexible quality of service model for mobile ad-hoc networks, in IEEE Vehicular Technology Conference (Tokyo, 2000), pp. 445-449

[11] C. Perkins, E. Belding-Royer, and S. Das, Ad hoc ondemand distance vector (AODV) routing, RFC 3561, July2003. 